\title{
Single Flap Upper Medial Vector Brachioplasty: A Modification to The Fascial Suspension Technique
}

\author{
AYMAN NOAMAN, M.D.; AHMED SAFWAT SALEM, M.D.; DINA BADAWY, M.D. and \\ MANAR KHATER, M.Sc.
}

The Department of General Surgery, Plastic Surgery Unit, Faculty of Medicine, Cairo University

\begin{abstract}
Background: Arm contouring for lipodystrophy and ptosis are increasing in demand with age and especially after massive weight loss following bariatric surgeries. Aesthetic arm contouring is one of the most requested surgeries to those patients. Multiple classification systems were described to guide the management during pre-operative assessment. Treatment options vary between liposuction only, skin resection only, a combination of liposuction and excision, or possibly the use of newer energy based skin tightening devices. One of the most undesirable sequelae is visible scarring either due to widening or migration of the scars. Location of the scar and elimination of fat deposits and excess skin are the ultimate concerns of patients seeking this surgery. The focus of this study modification has been aimed to improve the poor aesthetic result of scar sequelae and over all arm contouring.
\end{abstract}

Aim of Work: Developing a modification in surgical technique for brachioplasty, that eliminates excess skin with adequate arm contouring, and position scars in the least visible location, thus achieving better overall patient satisfaction.

Methodology: This study was conducted on 15 patients who presented seeking arm contouring in the period from October 2017 to May 2019. Among these patients the average age was 38 . Average BMI was $27.5 \mathrm{~kg} / \mathrm{m}^{2} .60 \%$ of the cases presented following Bariatric surgeries, $26.7 \%$ presented with skin excess following fluctuations in weight and effect of aging, and $13.3 \%$ presented with excess skin following previous unsatisfactory liposuction.

Routine liposuction done initially, incision done down to the honeycomb plane, undermining was carried deep to the superficial fascia up to the anterior border of triceps. Skin flap was redraped and pulled in an upper medial vector.

Results: This upper medial vector helped in improving the contour of the arm in a homogenous way, eliminating skin excess especially in the area above the olecranon in the distal arm and also avoids the presence of bands while closure. Early complications was limited to one case with $2 \mathrm{~cm}$ wound dehiscence, while late complications showed 5 cases with scar hypertrophy and 4 cases with scar pigmentation.

Conclusion: The mentioned technique proved to be reliable in achieving favorable arm contouring, with acceptable scarring and a low incidence of complications.
Key Words: Arm contouring - Vector - Redundancy - Scar location.

\section{INTRODUCTION}

Arm contouring for lipodystrophy and ptosis are increasing in demand with age and especially after massive weight loss following bariatric surgeries where aesthetic arm contouring is one of the most requested surgeries to those patients [1].

Various deformities can occur in the arm either with excess skin laxity or excess and heterogeneous fat deposition, multiple classification systems were described to give a proper assessment to those patients, which include assessment of fat excess, skin excess, and location of the deformity (proximal arm, entire arm, and extension to lateral chest wall).

Management to those cases varies between liposuction, skin excision or combination of liposuction and skin resection, or possibly the use of newer energy based skin tightening devices [2]. Multiple methods had been described for arm rejuvenation, yet none of them has provided full satisfaction to both the patients and surgeons; among these methods are excess skin resection of the arm with the scar oriented horizontally placed in the brachial sulcus, W-plasties, and T closure and the de-epithelialized rolled-up flap, fascial suspension technique, and lipoaspiration. The focus of the modifications has been mainly to improve the aesthetic result of the scar and reduce possible complications [3].

One of the most undesirable sequelae is visible scarring either due to widening or migration of the scars. An ongoing, unresolved debate regards the optimal placement of the scar, with some authors supporting a posterior arm location, and others favoring medial placement in the bicipital groove 
also the most recently reported poster-medial scar [4].

Another issue that concerns patients seeking arm contouring is the presence of undesirable fat deposits, especially in the distal arm above the olecranon [5].

\section{PATIENTS AND METHODS}

This study was conducted on 15 patients who presented seeking arm contouring in the period from October 2017 to May 2019.

Demographic information, including age, sex, associated comorbidities, method and rate of weight loss and current body mass index, were gathered from all patients enrolled in the study.

A detailed pre-operative counseling with consents was done to all patients who were part of the study. The consent included the proposed treatment options, details of the pre-operative pictures, markings, proposed surgical intervention, scars position and possible post-operative complications. Patients approved enrolment in the study and publication.

Standardized photographs were taken with the patient's arm abducted at 90 degrees, using a $50 \mathrm{~mm}$ lens at $1 \mathrm{~m}$. photographs were taken preoperatively and at each follow-up visit, at 2 weeks, 1 month and 3 months.

\section{Inclusion criteria were as follows:}

- Individuals between the age of 20-50 years, presenting for arm contouring requiring surgical excision.

- Body mass index less than or equal to $30 \mathrm{~kg} / \mathrm{m}^{2}$.

- Minimum one year following bariatric surgery and 6-months of weight stabilization.

\section{Exclusion criteria include:}

- Patients with severe uncontrolled comorbidities that may limit surgery and affect proper healing.

- Patients presenting with arm lipodystrophy requiring liposuction only.

- Patients presenting with excess skin along the chest and upper body roll that will require upper body lift.

- Previous history of deep vein thrombosis and/or pulmonary embolism.

- Heamoglobin value less than $10 \mathrm{~g} / \mathrm{dl}$.

- Smoking habit (>5 cigarettes a day).

\section{Pre-operative markings:}

With the patient standing and the arm abducted at 90 degrees, a horizontal line was drawn along the bicipital groove extending from the axillary crease proximally till a point two fingers above the medial epicondyle; another line was drawn 1 finger breadth posteriorly which is the site of the incision to end in a postero-medial scar. A perpendicular extension of the line is marked along the axillary crease posteriorly, marking the modified scar placement along the axillary crease. The decision of the length of both the distal incision at the medial epicondyle and the proximal posterior extension along the axillary crease were kept for intra-operative decision according to the extent of the excision of the skin excess; with no extension beyond the elbow in the horizontal element of the scar, and the posterior axillary fold as the cutoff point that the axillary crease incision doesn't extend beyond, to avoid visibility of the scar. Areas requiring liposuction were marked as well in standing position with arm abducted and adducted.

\section{Surgical technique:}

General anesthesia was used in all patients. Procedure started with tumescence infusion for liposuction. The Tumescence was composed of $20 \mathrm{cc}$ of $0.1 \%$ lidocaine in an epinephrine/saline 1:1000,000 concentration. The liposuction was performed deep to the superficial fascia and addressed the entire circumference of the arm with a special concern on the proximal postero-lateral aspect of the arm, the planned flap to be resected and distally at the area above the olecranon. The procedure ended with superficial liposuction to eliminate irregularities and to enhance skin retraction.

An incision was placed at the previously drawn line posterior to the bicipital groove; the incision was then deepened to the honeycomb plane that was created by the liposuction cannula. Dissection proceeded inferiorly to the desired extent which always reached the anterior border of the triceps muscle with undermining between the superficial fascia of the skin and the deep fascia of the muscle.

The undermined flap was then pulled completely in an upper medial vector towards the axillary crease, this vectors helps eliminate excess skin especially at the elbow in a homogenous way, and prevents the presence of bands. Excess skin was excised in a tailor tucking manner from distal to proximal to prevent any risk of skin over resection. The fascia of the undermined flap was tacked and fixed to the deep axillary fascia in the axillary crease with multiple interrupted 2/0 PDS sutures 
in the same fascial suspension technique previously described by Elkhatib in 2013 [3]. The upper medial vector pull of the skin flap directs the excess dog ears towards the axillary crease marking which was planned pre-operatively. Excess skin can be removed along the axillary crease incision directed posteriorly as an L-shaped scar or with a small Tscar if a small extension anteriorly is needed along the axillary crease. Layered closure was performed with absorbable sutures. Compression garments were instructed to be used by all patients for 6 weeks post-operatively.
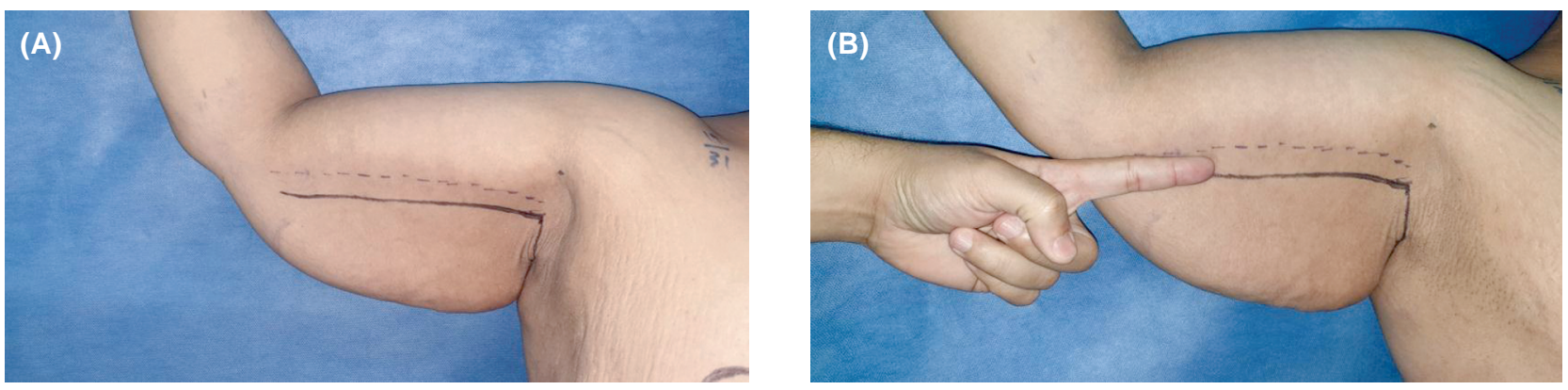

Fig. (1): Pre-operative marking where the arm is abducted 90 degrees, the above dotted line is at the bicipital groove (A) while the solid line is one finger breadth posteriorly (B), and also there is another line along the axillary crease.
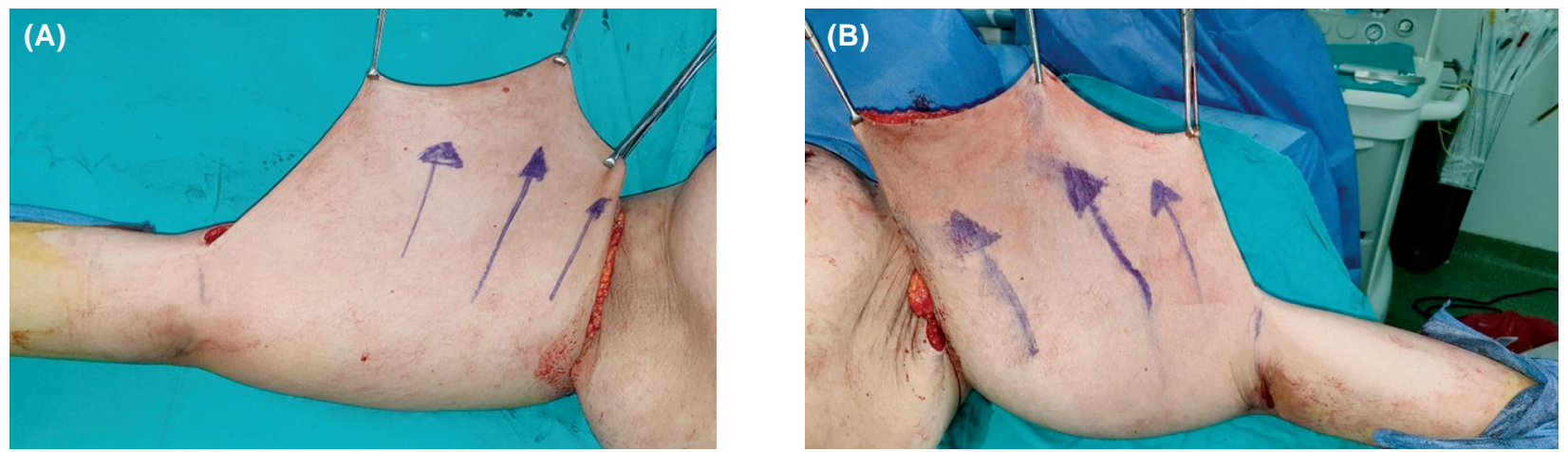

Fig. (2): Showing pulling the undermined flap in an upper medial vector towards the axilla, (A) the right arm and (B) the left arm.

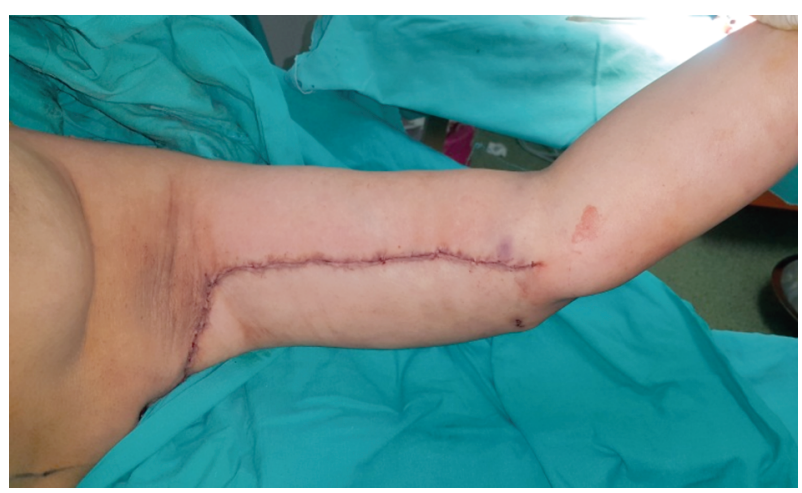

Fig. (3): Immediate post-operative picture after closure of the wound.

\section{RESULTS}

Fifteen patients underwent brachioplasty with the described technique in this study. Nine patients had bariatric surgeries with massive weight loss, four patients complained of arm redundancy as a consequence of weight fluctuation and aging, and two patients complained of excess arm skin laxity after a previous procedure of liposuction. Mean age at of the patients in the study was 38 years (range between 24 to 57 years). The mean body mass index was $27.5 \mathrm{~kg} / \mathrm{m}^{2}$ (range between 24 to $\left.30 \mathrm{~kg} / \mathrm{m}^{2}\right)$.

For early complications; one patient had a 2$\mathrm{cm}$ wound dehiscence at the junction of the horizontal and vertical wounds and was treated in outpatient setting through repeated dressing and follow-up. Neither thromboembolic events nor major systemic complications were experienced. No hematoma, seroma, infection or skin necrosis occurred in any of the patients.

For late complications; five patients experienced hypertrophied scar and were treated with topical therapy (silicone gel sheets), four patients complained of scar pigmentation and were instructed to use skin lightening creams (containing both Vitamin $\mathrm{C}$ and glutathione). Neither scar widening nor axillary contractures nor lymphedema presented as complains in this study. 

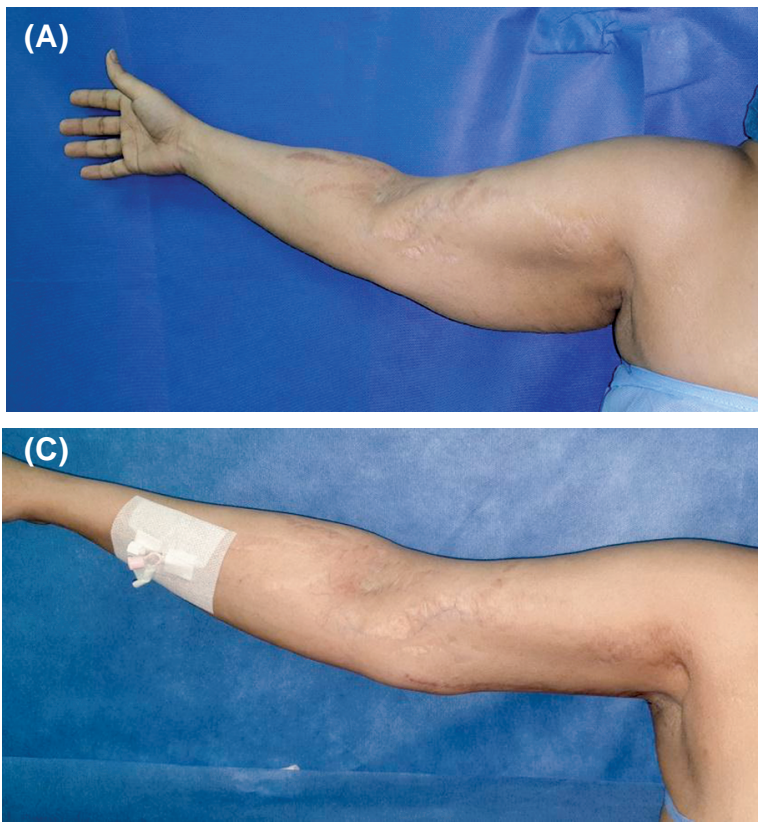

Fig. (4): A 42y old female patient where (A \& B) are pre-operative photos while (C \& D) are 6 months post-operative.
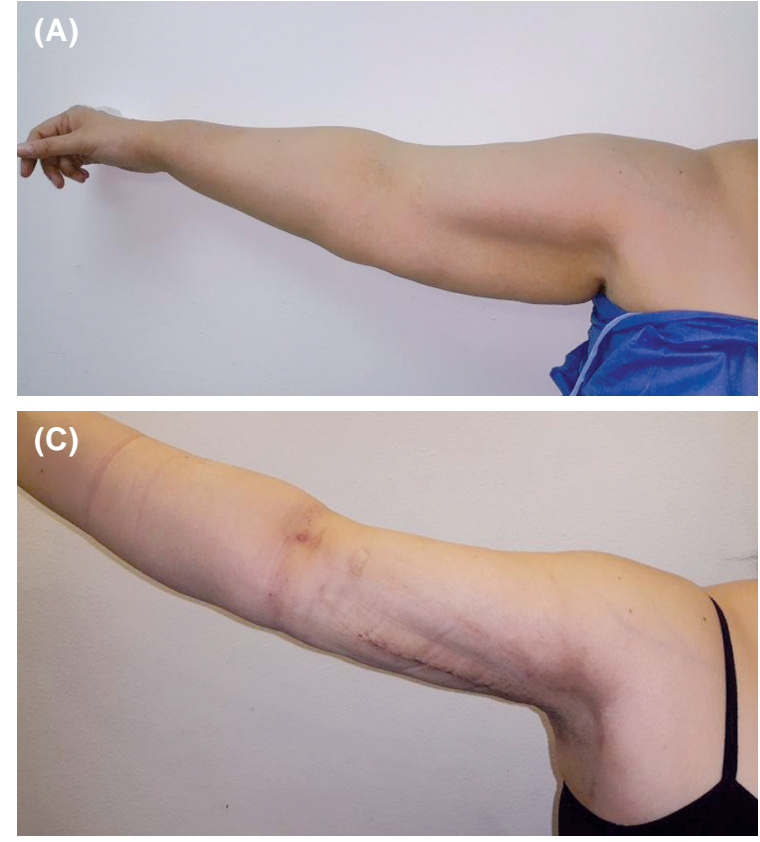

Fig. (5): A 25y old female patient where (A \& B) are pre-operative photos while (C \& D) are 4 weeks post-operative.

\section{DISCUSSION}

Brachioplasty was first described in 1930s as a reduction procedure of pendulous arms in obese women. However, the first aesthetic brachioplasty was described by Correa-Iturraspe and Fernandez in the 1950 s, and since that time, brachioplasty has become a standard procedure for upper limb aesthetic contouring. The most frequent causes leading to arm redundancy are the natural aging process, massive weight loss due to diet and exer-
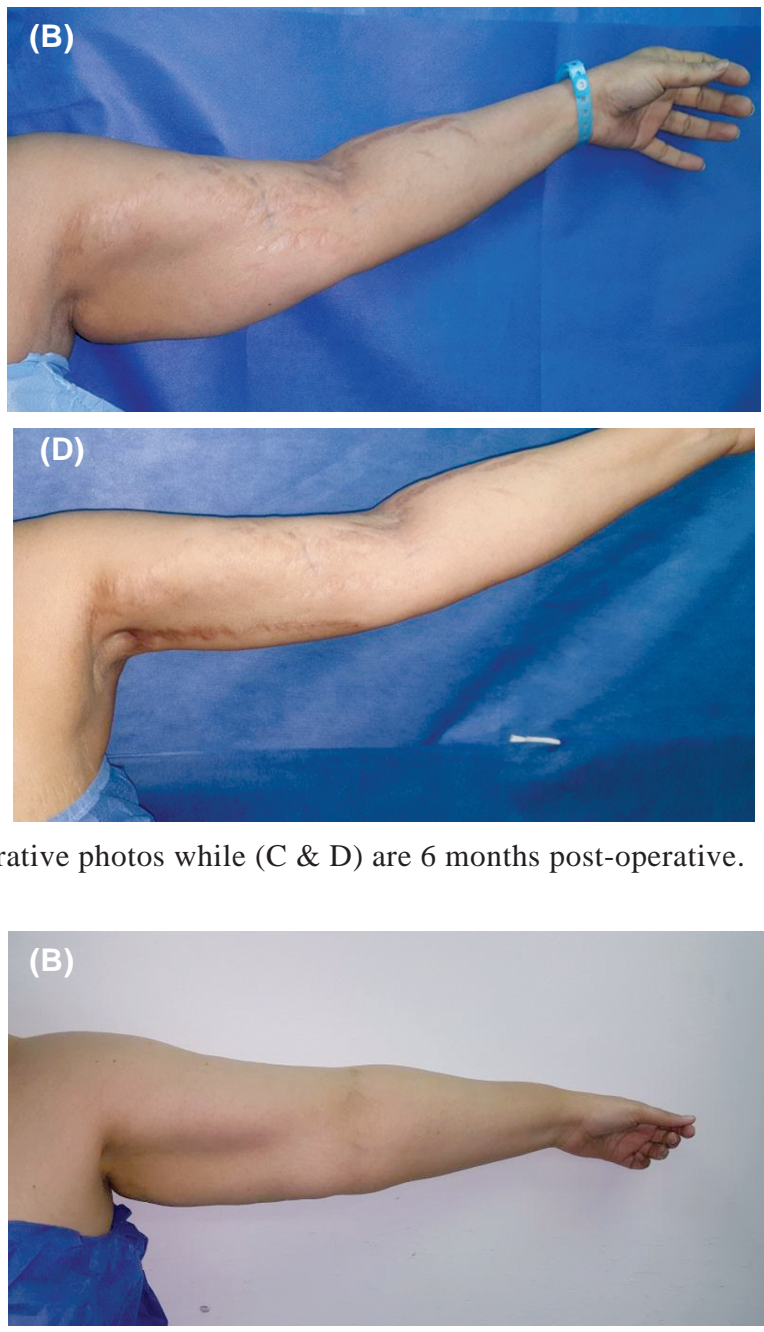

(D)

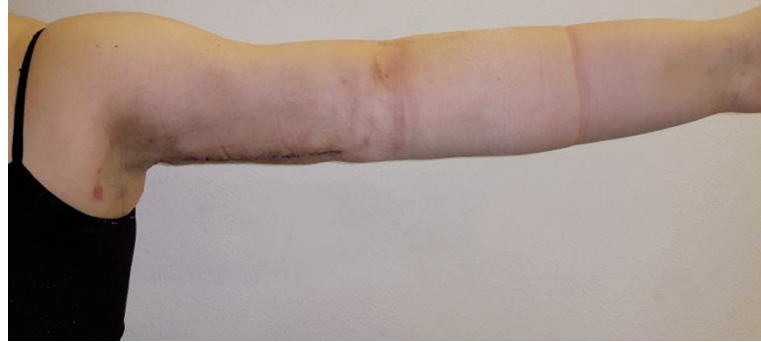

cise, and weight loss following bariatric surgeries [6].

The number of performed brachioplasty procedures increased in the past decade, for both massive weight loss patients (39.8 percent) and the general population (60.2 percent) as reported by the American society of aesthetic surgery [7].

Despite being an effective treatment for arm contouring, brachioplasty is associated with signif- 
icant complication ( 25 to 40 percent) and revision rates (3 to 25 percent) which can be considered relatively high for an elective aesthetic procedure. Commonly presented complications include widened hypertrophic scar, visible scar, residual skin laxity, wound dehiscence, and wound infection [8].

However, there is still controversy about what would be the optimal location of the scar. In 2013, Samra et al., surveyed plastic surgeons, the general population, and former brachioplasty patients about the optimal scar location. They found that the most aesthetically acceptable location is the medially placed scar [9].

The posteromedial scar approach is not visible from behind, and compared with the classic medial location, it is less visible from the frontal view while the patient is abducting his/her arms [10] and this is why it was the incision of choice in this study.

Routine liposuction helped in dissecting the flap between the superficial fascia and the deep fascia of the muscle beneath, such technique was also carried out by Nguyen and Rohrich, 2010 in their work. Undermining the posterior flap till the anterior border of the triceps muscle or further posterior carried no risk since it was limited to a plane between the superficial fascia and deep fascia of the arm, this was evident also in the work of Shermak, 2014. Such techniques reduced possible risk of affecting skin vascularity [13,14]. No patients suffered from skin necrosis or sloughing in the study.

El-Khatib proposed a modification by adding suspension of the anterior flap to the deep fascia in an attempt to prevent tension at the suture line thus improve scar quality, and reduce scar migration in turn less visibility $[2,3]$. This is why in this study we combined the idea of the postero-medial scar with fascial suspension technique to prevent migration or widening of the scars.

The idea of the pulling the undermined flap in an upper medial vector, and placing the scar along the axillary crease in a posterior direction, was to limit the scar from crossing the axilla, for two reasons, the first was to prevent the extension of the scar on the chest wall reducing scar visibility in non-indicated patients, and the second was to prevent axillary contracture. Many patterns were previously described including straight line scar, a "T" shape scar, "W" shape, an "L" shape, and "S" shape scar and sometimes including a Z-plasty in the axilla to prevent contractures [11,12].
This upper medial vector helped in improving the contour of the arm in a homogenous way, eliminating skin excess especially in the area above the olecranon in the distal arm and also avoids the presence of bands while closure. The tailor tucking excision of the skin prevented over resection of the skin excess.

\section{Conclusion:}

The mentioned technique proved to be reliable in achieving favorable arm contouring, with acceptable scarring and a low incidence of complications. The upper medial vector helped eliminate excess skin, improve the contour of the arm in a homogenous way and place the scar in a concealed location.

Combining the postero-medial scar acceptance to patients, with benefits of the fascial suspension technique and the tailor tacking excision of the skin helped reduce complications and overall patient satisfaction.

\section{Declaration of conflicting interests:}

The authors declared no potential conflict of interest with respect to the research, authorship, and/or publication of this article.

\section{Acknowledgement: None.}

\section{Funding:}

The authors did not receive any funding for the completion of this work.

\section{Informed consent:}

A detailed informed consent was obtained from all patients who were part of the study. The consent included details of the pre-operative pictures, measurements, method of surgical intervention, scars position and possible post-operative complications. Patients approved enrolment in the study and publication.

\section{REFERENCES}

1- Aly A., Pace D. and Cram A.: Brachioplasty in the patient with massive weight loss. Aesthet. Surg. J., Jan.-Feb., 26 (1): 76-84, 2006.

2- Gabriele Miotto, M.D., M.Ed.; and Yan Ortiz-Pomales, M.D., F.A.C.S.: Arm Contouring: Review and Current Concept: Aesthet. Surg. J., Jul. 13, 38 (8): 850-60, 2018.

3- Hamdy Elkhatib, M.D.: Posterior Scar Brachioplasty with Fascial Suspension: A Long-term Follow-up of a Modified Technique: Plast. Reconstr. Surg. Glob. Open, Oct., 7; 1 (6): e38, 2013.

4- Simone P., Carusi C., Segreto F., Iannuzzi R., Buscaglione S., Gizzi A., Giannitelli S., Rainer A., Filippi S. and Persichetti P.: Postbariatric Brachioplasty with Postero- 
medial Scar: Physical Model, Technical Refinements, and Clinical Outcomes. Plast. Reconstr. Surg., Feb., 141 (2): 344-53, 2018

5- Hill S., Small K.H., Pezeshk R.A. and Rohrich R.J.: Liposuction-Assisted Short-Scar Brachioplasty: Technical Highlights. Plast. Reconstr. Surg., Sep., 138 (3): 447e50e, 2016.

6- Zomerlei T.A., Neaman K.C., Armstrong S.D., Aitken M.E., Cullen W.T., Ford R.D., Renucci J.D. and VanderWoude D.L.: Brachioplasty outcomes: A review of a multipractice cohort. Plast. Reconstr. Surg., Apr., 131 (4): 883-9, 2013.

7- American Society of Plastic Surgeons. 2015 plastic surgery statistics report. Available at: https://www.plasticsurgery. org/news/plastic-surgery-statistics. Accessed October 10, 2016.

8- Andrea Sisti, Roberto Cuomo, Luca Milonia, Juri Tassinari, Antonio Castagna, Cesare Brandi, Luca Grimaldi, Carlo D'Aniello and Giuseppe Nisi: Complications associated with brachioplasty: A literature review Acta Biomed, Jan. 16, 88 (4): 393-402, 2018.
9- Samra S., Samra F., Liu Y.J., Sawh-Martinez R. and Persing J.: Optimal placement of a brachioplasty scar: A survey evaluation. Ann. Plast. Surg., 71 (4): 329-32, 2013.

10- Knotts C.D., Kortesis B.G. and Hunstad J.P.: Avulsion brachioplasty: Technique overview and 5-year experience. Plast. Reconstr. Surg., 133: 283-8, 2014.

11- Appelt E.A., Janis J.E. and Rohrich R.J.: An algorithmic approach to upper arm contouring. Plast. Reconstr. Surg., 118 (1): 237-46, 2006.

12- Pascal J.F. and Le Louarn C.: Brachioplasty. Aesthetic Plast. Surg., 29 (5): 423-9; discussion 430, 2005.

13- Nguyen A.T. and Rohrich R.J.: Liposuction-assisted posterior brachioplasty: Technical refinements in upper arm contouring. Plast. Reconstr. Surg., 126 (4): 1365-9, 2010.

14- Shermak M.A.: Aesthetic refinements in body contouring in the massive weight loss patient: Part 2. Arms. Plast. Reconstr. Surg., 134 (5): 726e-735e, 2014. 